\title{
Multi-software-hybrid-programming digital image processing for high definition single lens digital imaging system
}

\author{
Chijun Li ${ }^{a}$ Xiang Ren ${ }^{b}$ Yule Huang ${ }^{c}$, Kai Yang ${ }^{d}$,Peiying Liang ${ }^{*}{ }^{*}$, Hui Cao ${ }^{*}$ \\ School of Electronic Information Engineering, Foshan University, Foshan 528000, China \\ School of Physics and Optoelectronic Engineering, Foshan University, Foshan 528000, China;

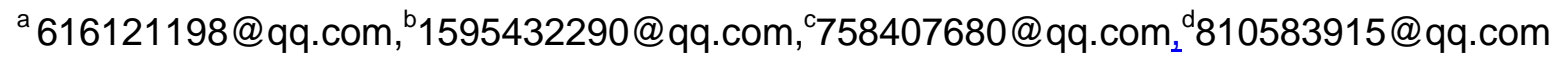 \\ ${ }^{*}$ Corresponding author: 13450809109@163.com \\ ${ }^{*}$ Corresponding author: foshancaohui@sina.com
}

Keywords: Digital Image Processing, hybrid programming, CMOS,single lens imaging system Abstract: We designed a multi-software-hybrid-programming digital image processing software for high definition single lens digital imaging system. In this digital image processing software system, LabVIEW ,MATLAB,Quartus ii and C++ are all employed to call DLL library to invoke the CMOS, read data, process image and improve the image quality. Such a software system is specially designed to extend the depth of field in single lens imaging system. Especially, one-button-operation function is designed to reduce the operating time. The results showed that with this software system, even in the large defocus case, the high definition and clear images are ready to output from the single lens imaging system. This system can be used in the fields of biological and medical microscopic imaging, machine vision, etc.

\section{Introduction}

In many cases, such as machine vision and biological imaging, even if only single lens is used, people also hope to get a large depth of field imaging. A digital image processing software for high definition single lens digital imaging system is indispensable.

Generally, the multi-software-hybrid-programming digital image processing for high definition single lens digital imaging system uses LabVIEW , andcalls DLL library to invoke the CMOS and the node of MATLAB for image processing. At the same time, the multi-software-hybrid-programming digital image processing for high definition single lens digital imaging system which comes with an image processing algorithm can improve the image quality, and can make the image more adaptable to the human standards of beauty. Such a hybrid software system can also achieve the aim of taking photos and processing images. However,the reported system has failed to cater for one-button operation to reduce operating time, and thus this software system are unsatisfactory. Not only should we improve the software system, but also we should implement one software processing, avoiding multiple software operation.

In this paper,we report a multi-software-hybrid-programming digital image processing for high definition single lens digital imaging system that is designed with one-button-operationfunction. This function not only can control the whole application, but also can improve the running speed of the application, and reduce the operating time.

\section{Multi-software hybrid program}

A digital image processing software for high definition single lens digital imaging system is designed by means of multi-softwarehybridprogramming. The user interface of the software is shown in Fig. 1. As is shown in Fig. 2, based on the application of LabVIEW, we call library function node, controlling the CMOS. We use the Quartus II to program the task to the chip,package the code to DLL,transfer it to LabVIEW.Especially,the DLL is packaged by $\mathrm{C}++$. Figure 3 presented the Codes of $\mathrm{C}++$. 


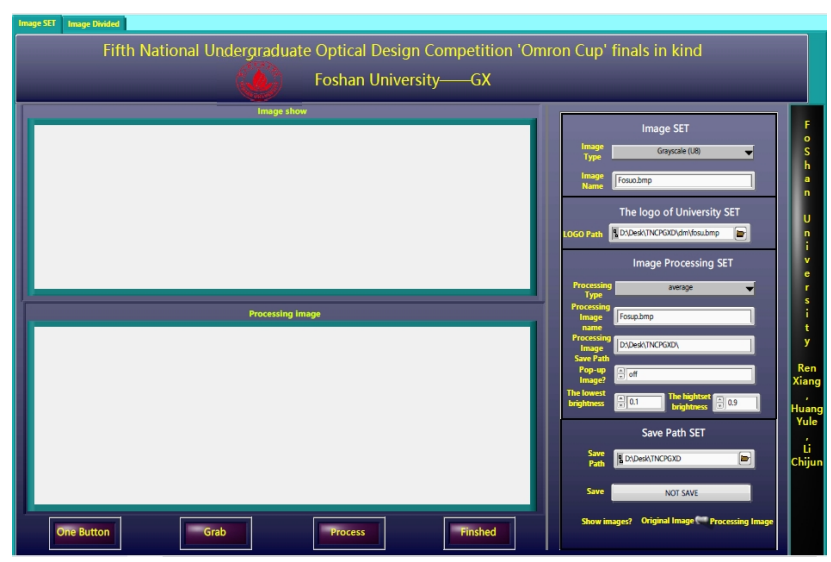

Fig.1 User interface

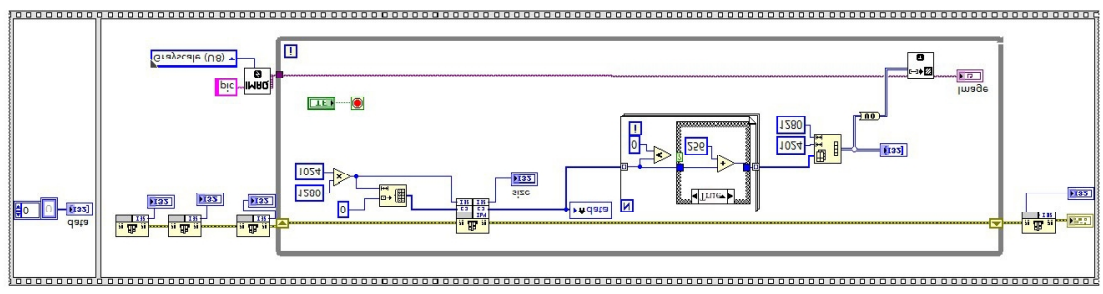

Fig. 2 The Codes forinvoking Camera of CMOS

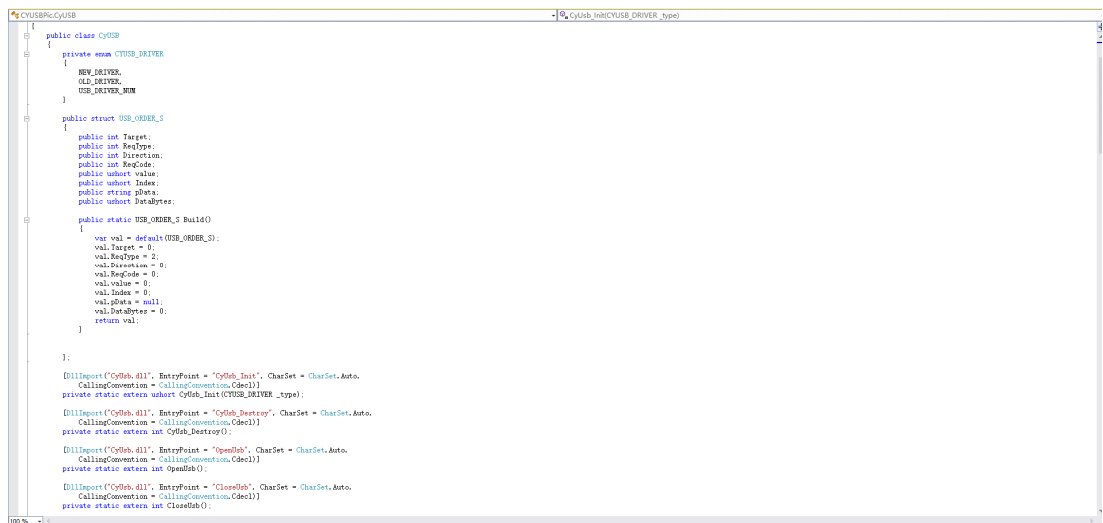

Fig.3 The Codes of $\mathrm{C}++$

Otherwise, in the application of LabVIEW, we use the MATLAB function to process images.

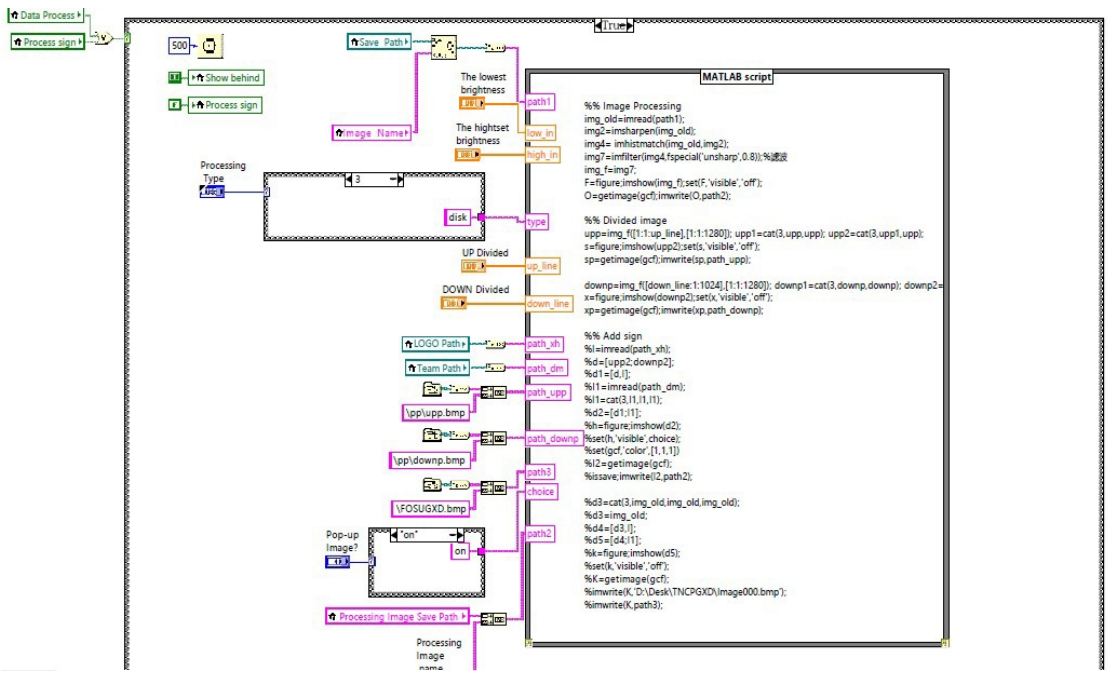

Fig. 4 Image Processing's Codes 
It should be pointed out that MATLABfunctions, including 'imadjust', 'histeq', 'imsharpen','adapthisteq', 'imhistmatch', 'decorrstretch' are used to process image and improve its quality. With the following steps, we use the gradation processing to adjust the brightness until meeting the comfortable needs of the human visual experience.

Firstly, we use the histogram specification function to enhance the overall contrast of the image automatically. As is well known, it's not easy to control specific enhancement, the result of the processing is always to result in a global uniform histogram. Here, we use the histogram equalization which is better than the histogram specification. ${ }^{[1]}$

Secondly,we also use histogram match to find the same thing of two images by matching the acquisition of high-quality map and diagram, and then enhance the percent of matching between two images. Compared to other dealing ways, it is more automatic,quicker,with higher accuracy. ${ }^{[2]}$

Thirdly,we use the Gaussian filter to deal with the image. the Gaussian matrix is as follows. ${ }^{[3]}$

$$
\left[\begin{array}{ccccc}
-1 & -4 & -7 & -4 & -1 \\
-4 & -16 & -26 & -16 & -4 \\
-7 & -26 & 505 & -26 & -7 \\
-4 & -16 & -26 & -16 & -4 \\
-1 & -4 & -7 & -4 & -1
\end{array}\right]
$$

In general, the Gauss filter is a process to result in the weighted average of the entire image. The gray value of each pixel is obtained by weighted average of the gray values of each pixel and the other neighboring pixels. Using a template (or convolution mask) to scan each pixel in the image, the gray value of the center pixel is determined by the weighted average of all the pixel in the template. Gauss algorithm can be used to improve the quality of the image to show the details of the ISO-12233 standard test chart.

What's more,the multi-software-hyborid-programming digital image processing for high definition single lens digital imaging system is designed with one-button-operationfunction and the function about dividing images. We use MATLAB to divide images by processing the matrix of the images.Therefore, we process all the data within the matrix about the images,changing every digital dot to the most suitable value. Figure 5 and Fig. 6 showed the original Picture and the divided Picture.

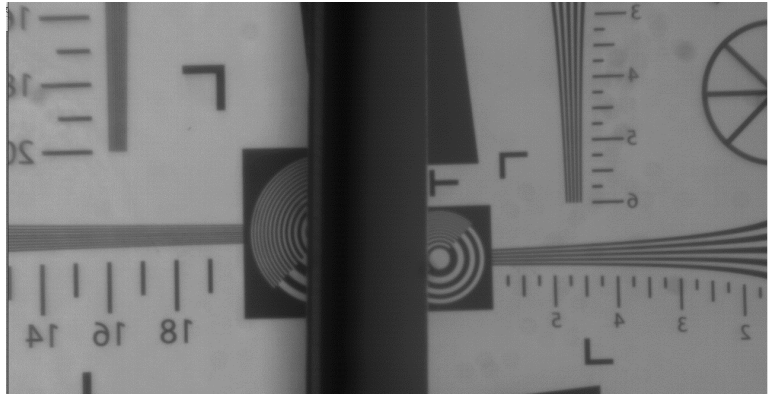

Fig.5 Original Picture

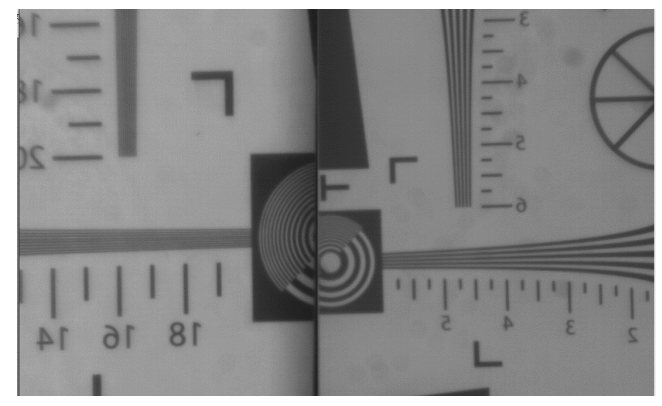

Fig.6 Divided Picture

\section{Experimental results and discussion}

The multi-software-hybrid-programming digital image processing softwareperfectlycombined with high definition single lens digital imaging system. Figure 7 and Fig. 8 showed the original picture and the processedpicture. The original resolution of 13-lines in ISO-12233 can promote to 14-lines, and improving the imaging effect greatly. ${ }^{[4]}$ It means that the multi-software-hybrid-programming digital image processing algorithms enable to improve the clarity and resolution of image. 


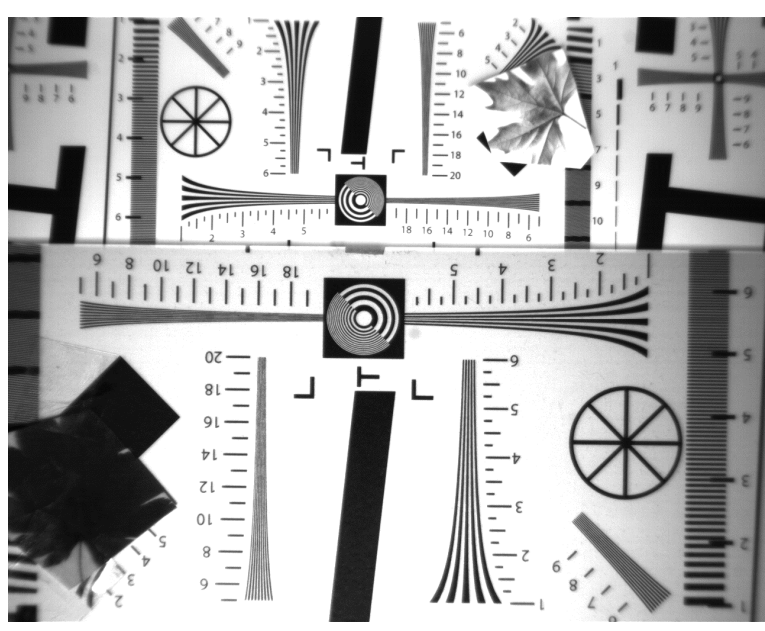

Fig.7 Original Picture

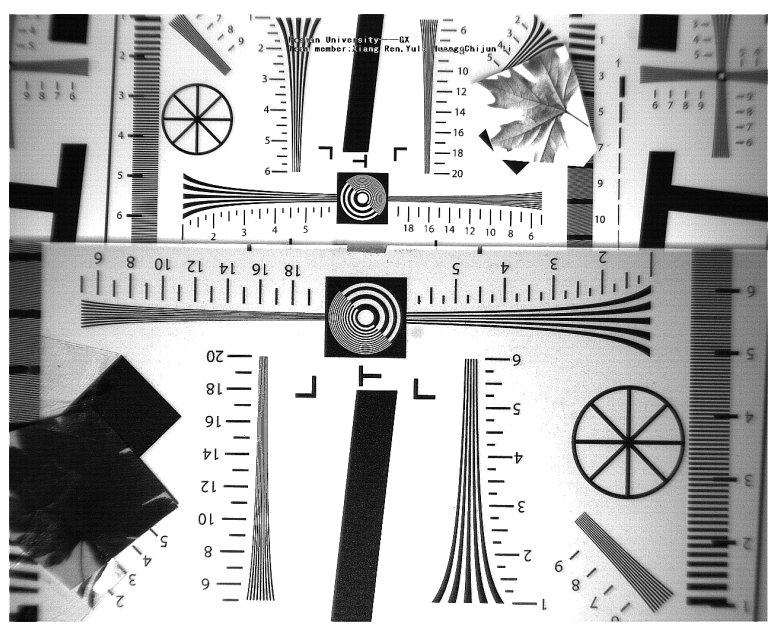

Fig.8 Processed Picture

The multi-software-hybrid-programming digital image processing for high definition single lens digital imaging system can be operated free and easily, matching high definition single lens digital imaging system. At the same time it can divided two pictures, deleting the part of blank.

In order to quantitatively analyze the image quality, softwareImatest version 3.4 was employed to evaluate the image. Fig. 9 and Fig.10 presented the software evaluation results of original picture and processed picture. For original Picture, it is $853.9 \mathrm{LW} / \mathrm{PH}$, which is lower than that of the processed picture $(1644 \mathrm{LW} / \mathrm{PH})$. It means the contrast and clarity of the quality of the processed image is obviously improved.
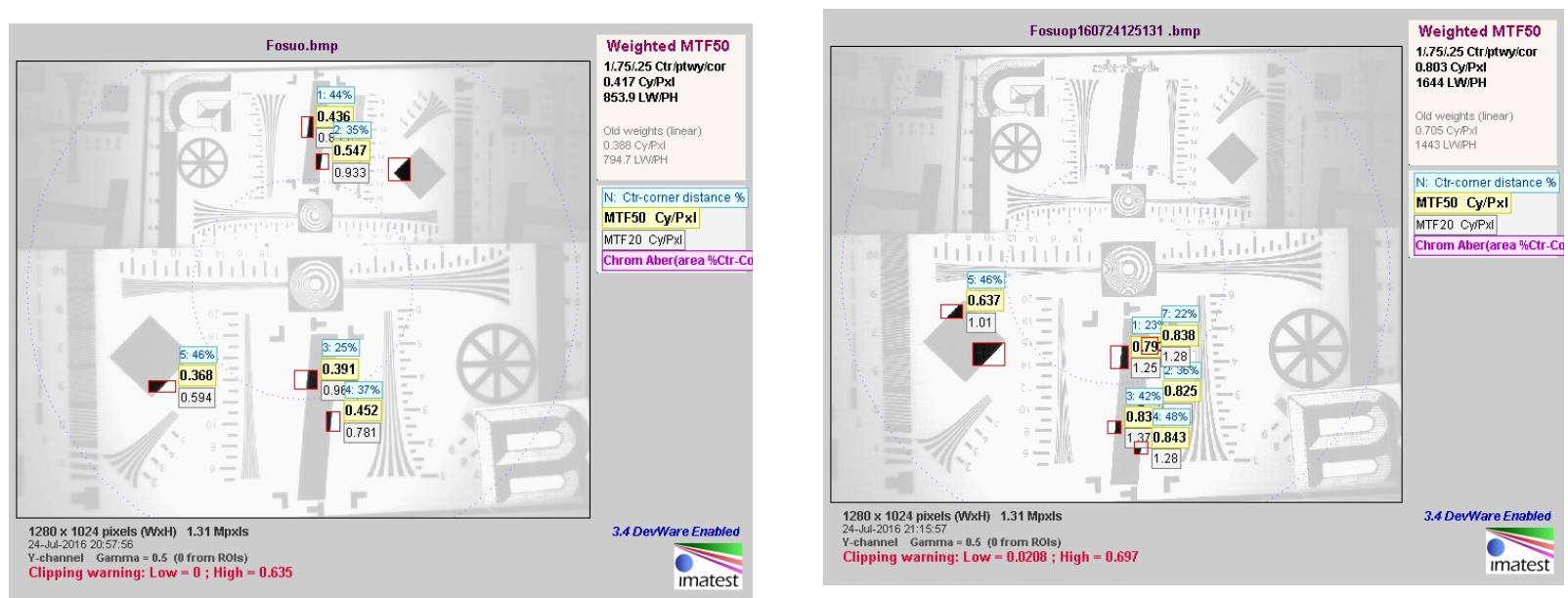

Fig.9 The Measurement data of original pictureFig.10 The measurement data of processed picture

\section{Conclusions}

We reported a multi-software-hybrid-programming digital image processing software for high definition single lens digital imaging system. In this software system, LabVIEW ,MATLAB,Quartus ii and $\mathrm{C}++$ are combined to call DLL library to invoke the CMOS, read data, process image and improve the image quality. Especially, one-button-operation function is designed to reduce the operating time. The results showed that with this software system, even in the large defocus case, the high definition and clear images are ready to output from the single lens imaging system. This system can be used in the fields of biological and medical microscopic imaging, machine vision, etc. 


\section{Acknowledgements}

This work was financially supported by the Natural Science Foundation of Guangdong Province, China (2015A030313633), Key Project of Department of Education of Guangdong Province (2014KTSCX153), Construction project of Foshan science and technology innovation platform(2016038), Fundamental Research Funds of Foshan University (2014042), Science and Technology Research \& Development Funds of Foshan(2012AA100451) and National Natural Science Foundation of China (NSFC) (61178030).

\section{References}

[1] Wang Ji and Lu Wen-Kai :Coherence cube enhancement based on local histogram specification [J], APPLIED GEOPHYSICS, Vol.7, No.3, P. 249 - 256, 5 Figures(September 2010)

[2] Ashish Ghosh; Badri Narayan Subudhi;Susmita Ghosh:Object Detection From Videos Captured by Moving Camera by Fuzzy Edge Incorporated Markov Random Field and Local Histogram Matching[J],IEEE TRANSACTIONS ON CIRCUITS AND SYSTEMS FOR VIDEO TECHNOLOGY, VOL. 22, NO. 8(AUGUST 2012)

[3] YetaiFei; Kuang-Chao Fan; RongshengLu:Decreasing the amp;otide deviation of Guassian filter in surface roughness measurements[J],SPIE 7130, Fourth International Symposium on Precision Mechanical Measurements, 713054 (31 December 2008);

[4] Lisa Wang,PuWan,Hao Kong[J]: Virtual camera lens depth of field effects improved algorithm simulation, Jilin Normal University Journal (2003) 